\title{
Fidelidade do "potential acuity meter" (PAM) no prognóstico da acuidade visual pós-operatória de cirurgia de catarata
}

\author{
Fidelity of the potential acuity meter in the postoperative visual acuity \\ of cataract surgery
}

\author{
Brenda Biagio Chiacchio ${ }^{1}$ \\ Ricardo MitsuoSato ${ }^{2}$ \\ Roberta Bianca Peres Siqueira ${ }^{3}$ \\ Frederico França Marques ${ }^{4}$
}

Trabalho realizado no Hospital Monumento e Complexo Hospitalar Padre Bento.

${ }^{1}$ Residente do Hospital Monumento e Complexo Hospitalar Padre Bento - São Paulo (SP) - Brasil.

2 Residente do Hospital Monumento e Complexo Hospitalar Padre Bento - São Paulo (SP) - Brasil.

${ }^{3}$ Residente do Hospital Monumento e Complexo Hospitalar Padre Bento - São Paulo (SP) - Brasil.

${ }^{4}$ Chefe do Setor de Catarata do Hospital Monumento e

Complexo Hospitalar Padre Bento - São Paulo (SP) -

Brasil.

Endereço para correspondência: Frederico F. Marques. Rua Arapá, 28 - Apto 31 - São Paulo (SP)

CEP 04363-060

E-mail: fredani2010@ hotmail.com

Recebido para publicação em 14.07.2007

Última versão recebida em 05.08.2008

Aprovação em 20.08.2008

\section{RESUMO}

Objetivo: Avaliar a relação da acuidade visual (AV) obtida pelo "potential acuity meter" (PAM) no pré-operatório de cirurgia de catarata com a acuidade visual obtida no pós-operatório, bem como, sua correlação com a classificação morfológica dominante da catarata. Métodos: Trata-se de um estudo prospectivo realizado no setor de Catarata do Centro de Estudos do Hospital Monumento envolvendo 63 olhos de 45 pacientes avaliados de julho a setembro de 2006, submetidos à cirurgia de catarata sob a técnica de facoemulsificação com implante de lente intra-ocular, sendo posteriormente excluído 1 olho. No período pré-operatório, foi realizado o PAM sob midríase e seu resultado foi comparado à melhor acuidade visual pósoperatória do terceiro mês e correlacionado com a classificação morfológica da catarata, sendo denominado satisfatório aquele resultado que não variou mais do que duas linhas na tabela de Snellen. Resultados: A média de idade foi de 45,3 anos com média da acuidade visual obtida pelo PAM de 0,64 $\log$ MAR. No terceiro mês pós-operatório, a média da melhor acuidade visual corrigida (MAVC) foi de 0,09 $\log$ MAR. O PAM hiperestimou o resultado da MAVC em 8 olhos (13\%), hipoestimou em 41 olhos (66\%) e nos 13 olhos restantes (21\%), as acuidades foram idênticas. Este apresentou uma fidelidade inversamente proporcional à intensidade de opacidade do cristalino no caso da catarata nuclear $(1+\operatorname{com} 75,5 \%$ e $4+$ com $33,3 \%$ ), entretanto esta acurácia foi maior nos casos de subcapsular posterior $(85,7 \%)$. Conclusão: O PAM hipoestimou ou manteve da acuidade visual na maioria dos casos (87\%). Apresentou uma fidelidade inversamente proporcional à intensidade de opacidade do cristalino no caso da catarata nuclear, $1+\operatorname{com} 75,5 \%$ e $4+\operatorname{com} 33,3 \%$; entretanto esta acurácia foi maior nos casos de subcapsular posterior $(85,7 \%)$.

Descritores: Extração de catarata; Acuidade visual/fisiologia; Testes de visão/métodos; Técnicas de diagnóstico oftalmológico/instrumentação

\section{INTRODUÇÃO}

O interesse nos fatores que contribuem para a avaliação pré-operatória na cirurgia de catarata tem crescido juntamente com o avanço de novas tecnologias nos facoemulsificadores e lentes intra-oculares reduzindo assim a incidência de complicações, por conseguinte, proporcionando uma reabilitação visual mais precoce. Sendo assim, quanto mais informações obtivermos ao exame pré-operatório, principalmente, o potencial de visão 
do paciente, maior será a chance de proporcionarmos uma real expectativa ao mesmo ${ }^{(1)}$. Além disso, a presença de comorbidades geralmente associadas pela idade avançada nos casos de catarata de etiologia senil torna ainda mais importante esta avaliação $0^{(2)}$.

O "potential acuity meter" (PAM) consiste na projeção de um feixe de luz com optotipos da tabela de Snellen na região macular, preferencialmente através de uma midríase, simulando o potencial de acuidade visual no período pós-operatório após a remoção sem intercorrências da catarata em um paciente sem outras afecções oculares ${ }^{(2-4)}$.

Este trabalho avalia a relação da acuidade visual obtida pelo PAM no período pré-operatório da cirurgia de catarata com a melhor acuidade visual obtida no pós-operatório, bem como, sua relação com a classificação morfológica dominante da catarata e sua intensidade de opacificação quando do tipo nuclear.

\section{MÉTODOS}

Trata-se de um estudo prospectivo realizado no setor de Catarata do Centro de Estudos do Hospital Monumento, envolvendo 63 olhos de 45 pacientes avaliados de julho a setembro de 2006, submetidos posteriormente à cirurgia de catarata sob a técnica de facoemulsificação com implante de lente intra-ocular.

Os critérios de inclusão foram pacientes com catarata, alfabetizados e com percepção de optotipo de 20/400 e que compreenderam o exame e realizaram o acompanhamento pós-operatório como indicado, por sua vez, os critérios de exclusão foram pacientes que não conseguiram realizar ou compreender o exame, cataratas brancas, complicações intra ou pós-operatórias, e pacientes que não compareceram à visita de terceiro mês pós-operatório.

No período pré-operatório, além do exame oftalmológico completo composto por medida da melhor acuidade visual, tonometria de aplanação, biomicroscopia de segmento anterior, mapeamento de retina, biometria, foi realizado também o PAM (Mentor O\&O, Inc. Norwell, MA) sob midríase. Visando correlacionar seus resultados com a classificação morfológica da catarata, optamos por não utilizar a classificação LOCS II ("Lens Opacities Classification System") (5) devido a sua complexidade e sim a classificamos pela sua morfologia dominante em eixo visual de forma subjetiva sempre pelo mesmo examinador para reduzir as variáveis da subjetividade entre examinadores. Em relação ao tipo morfológico nuclear este foi classificado de $1+$ a $4+$ de acordo com a intensidade de sua coloração, sendo levemente amarelado como 1+ até brunescente como 4+.

Após a cirurgia, os pacientes foram examinados no primeiro e sétimo dia e no primeiro e terceiro mês, sendo realizado exame oftalmológico completo e os dados da melhor acuidade visual obtidos no terceiro mês foram comparados aos dados obtidos com o PAM e em seguida relacionados com a classificação morfológica da catarata.
Transformamos a AV em logMAR para comparação com a literatura.

Não se aplicou teste estatístico, pois a finalidade era tão somente verificar a porcentagem de resultados considerados satisfatórios ou insatisfatórios, entende-se casos satisfatórios aqueles cujos resultados pós-cirúrgicos de acuidade visual não variaram mais do que duas linhas na tabela de Snellen quando comparados ao resultado do PAM, e os insatisfatórios os demais casos. O teste de Qui-quadrado (X2) não foi utilizado devido à presença das restrições de Cochram.

\section{RESULTADOS}

Foram estudados 63 olhos de 45 pacientes com média de idade de 45,3 anos (variando 23 a 82 anos) sendo 23 homens $(51,1 \%)$ e 22 mulheres $(48,9 \%)$ e destes excluído 1 olho pois apresentou no pós-operatório edema macular cistóide. A média da acuidade visual obtida com o PAM no período préoperatório foi de 0,64 $\log \mathrm{MAR}$ (variando 1 a $3 \log \mathrm{MAR}$ ), por sua vez, no pós-operatório de terceiro mês a média da melhor acuidade visual foi de $0,09 \log$ MAR (variando 0 a 0,7 ).

O PAM hiperestimou o resultado da melhor acuidade visual final em 8 olhos (13\%), hipoestimou em 41 olhos (66\%) e nos 13 olhos restantes (21\%), a acuidade visual pelo PAM e a pós-operatória foram idênticas (Tabela 1).

No grupo de olhos nos quais o PAM hiperestimou a acuidade visual final, os resultados satisfatórios foram de $75 \%$. Neste grupo, 4 dos 8 olhos apresentaram outras comorbidades prévias, entre elas, ambliopia, degeneração macular relacionada à idade, e retinopatia diabética proliferativa (Tabela 2). Por sua vez, nos olhos nos quais o PAM hipoestimou a acuidade visual final, somente $56 \%$ foram resultados satisfatórios.

Ao analisarmos a classificação morfológica dominante em eixo visual encontramos 52 olhos $(83,8 \%)$ com cataratas nucleares, 7 olhos $(11,3 \%)$ com subcapsular posterior, 2 olhos $(3,2 \%)$ com polar posterior e 1 olho $(1,6 \%)$ com catarata capsular anterior, apresentando resultados satisfatórios em 63,5\%, $85,7 \%, 100 \%$ e $100 \%$, respectivamente (Tabela 3 ).

Dentro do grupo de catarata nuclear, houve uma redução consistente dos resultados satisfatórios do PAM de acordo com a gravidade da opacidade, sendo $76,5 \%, 60,9 \%$, para cataratas nucleares $1+, 2+$ e $44,4 \%$ e $33,3 \%$ para cataratas nucleares $3+\mathrm{e} 4+$, respectivamente (Tabela 4). Nos olhos com catarata subcapsular posterior, o PAM revelou uma média de 0,14 $\log$ MAR (variando de 0 a $0,48 \log$ MAR), já a média da melhor acuidade visual no terceiro mês foi de 0,02 $\log$ MAR (variando de 0 a $0,18 \log$ MAR).

Tabela 1. Relação do PAM com a acuidade visual final

\begin{tabular}{lc} 
Acuidade visual final & Número de olhos (\%) \\
Hiperestimou & $8(13 \%)$ \\
lgual & $13(21 \%)$ \\
Hipoestimou & $41(66 \%)$ \\
\hline
\end{tabular}




\begin{tabular}{|c|c|c|c|}
\hline Paciente & $\begin{array}{c}\text { PAM } \\
(\log M A R)\end{array}$ & $\begin{array}{c}\text { MAV } 3^{\circ} \text { mês } \\
\text { (logMAR) }\end{array}$ & Comorbidade \\
\hline 1 & 0,18 & 0 & $\mathrm{Ndn}$ \\
\hline 2 & 0,30 & 0 & $\mathrm{Ndn}$ \\
\hline 3 & 0,30 & 0,10 & $\mathrm{Ndn}$ \\
\hline 4 & 0,30 & 0 & $\mathrm{Ndn}$ \\
\hline 5 & 0,30 & 0 & $\mathrm{Ndn}$ \\
\hline 6 & 0,10 & 0,30 & Ret. diab. proliferativa \\
\hline 7 & 0,30 & 0 & $\mathrm{Ndn}$ \\
\hline 8 & 0,10 & 0,18 & $\mathrm{Ndn}$ \\
\hline 9 & 0,40 & 0,70 & Ambliopia \\
\hline 10 & 0,60 & 0,30 & $\mathrm{Ndn}$ \\
\hline 11 & 0 & 0 & Ndn \\
\hline 12 & 0 & 0 & $\mathrm{Ndn}$ \\
\hline 13 & 0,18 & 0 & Ndn \\
\hline 14 & 0,10 & 0 & Ndn \\
\hline 15 & 0,54 & 0 & Ndn \\
\hline 16 & 0,18 & 0 & Ndn \\
\hline 17 & 0 & 0,18 & Ndn \\
\hline 18 & 0 & 0,10 & Ndn \\
\hline 19 & 0,18 & 0 & Ndn \\
\hline 20 & 0,18 & 0 & $\mathrm{Ndn}$ \\
\hline 21 & 0 & 0 & Ndn \\
\hline 22 & 0,10 & 0 & $\mathrm{Ndn}$ \\
\hline 23 & 0 & 0 & Ndn \\
\hline 24 & 0,60 & 0 & Ndn \\
\hline 25 & 0 & 0 & Ndn \\
\hline 26 & 0 & 0 & Ndn \\
\hline 27 & 0,10 & 0 & Ndn \\
\hline 28 & 0,70 & 0,70 & Adenoca. hipófise \\
\hline 29 & 0,40 & 0,10 & Ndn \\
\hline 30 & 0,18 & 0,10 & $\mathrm{Ndn}$ \\
\hline 31 & 0,10 & 0,10 & Ndn \\
\hline 32 & 0,30 & 0 & Ndn \\
\hline 33 & 0 & 0,10 & Ndn \\
\hline 34 & 0,18 & 0,18 & Ndn \\
\hline 35 & 0,10 & 0,40 & DMRI \\
\hline 36 & 0,54 & 0,10 & Ndn \\
\hline 37 & 0,30 & 0,30 & Ndn \\
\hline 38 & 1,00 & 0,48 & D. retina (Alto míope) \\
\hline 39 & 0,18 & 0,10 & Ndn \\
\hline 40 & 0,40 & 0 & Ndn \\
\hline 41 & 0,40 & 0 & Ndn \\
\hline 42 & 0,40 & 0 & Ndn \\
\hline 43 & 0,30 & 0 & Ndn \\
\hline 44 & 0,10 & 0 & Ndn \\
\hline 45 & 0,30 & 0 & Ndn \\
\hline 46 & 0,30 & 0,10 & Ndn \\
\hline 47 & 0,18 & 0 & Ndn \\
\hline 48 & 0,10 & 0 & Ndn \\
\hline 49 & 0,18 & 0 & Ndn \\
\hline 50 & 0,30 & 0 & $\mathrm{Ndn}$ \\
\hline 51 & 0,30 & 0,40 & Ret. diab. proliferativa \\
\hline 52 & 0,18 & 0,10 & Ndn \\
\hline 53 & 0,30 & 0,18 & Ndn \\
\hline 54 & 0,18 & 0,10 & Ndn \\
\hline 55 & 0,18 & 0 & Ndn \\
\hline 56 & 0 & 0 & Ndn \\
\hline 57 & 0 & 0 & Ndn \\
\hline 58 & 0,10 & 0 & Ndn \\
\hline 59 & 0,10 & 0 & Ndn \\
\hline 60 & 0,18 & 0,18 & Ndn \\
\hline 61 & 0,48 & 0 & Ndn \\
\hline 62 & 0,18 & 0 & Ndn \\
\hline \multicolumn{4}{|c|}{ 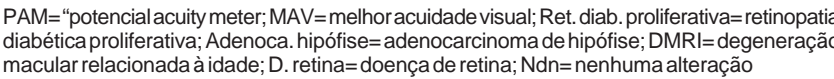 } \\
\hline
\end{tabular}

Nos dois olhos com catarata polar posterior a média do PAM foi de $0,18 \log$ MAR em contraste com $0 \log$ MAR obtido no pós-operatório. Por fim, no olho com catarata capsular anterior o PAM hipoestimou de 0,48 $\log$ MAR para 0,18 $\log$ MAR sendo o pós-operatório de $0 \log$ MAR.

\section{DISCUSS ÃO}

O PAM é considerado um bom método para predição da acuidade visual pós-operatória, principalmente em cataratas ainda não muito densas ${ }^{(3-4,6-8)}$.

O número de casos satisfatórios encontrados em nosso estudo foi de $67,7 \%$ em contraste com descrito na literatura que relata um resultado satisfatório do PAM em $100 \%$ dos casos quando a variação foi de até 3 linhas em acuidade visual préoperatória melhor ou igual a 20/200; e de $91 \%$ considerandose duas linhas de variação na tabela de Snellen ${ }^{(4)}$. Esta diferença pode ser resultante de uma variável não estudada pelos autores que é a classificação morfológica dominante, podemos perceber uma consistente redução de sua acurácia de acordo com a gravidade da opacidade, o que ficou demonstrado ao analisarmos a relação do PAM com as diferentes densidades da catarata nuclear, variando de 76,5\% (1+) para 33,3\% (4+).

Ao analisarmos os casos de hiperestimação do resultado do PAM; em um estudo de 137 olhos utilizando a visão interferencial com franjas a laser esta apresentou associação com doenças oculares como descolamento seroso de retina, edema macular cistóide, ambliopia, e degeneração macular relacionada à ida$\mathrm{de}^{(9)}$. Estas alterações foram encontradas em 4 dos 62 olhos.

O PAM apresenta algumas limitações, como uma boa compreensão por parte do paciente a ser examinado e a necessidade de uma transparência relativa de meios ${ }^{(4)}$, não sendo portanto um bom método em catarata brancas ou "nigrans". Entretanto, sua utilização como exame pré-operatório apresenta a função de revelar apenas uma estimativa de acuidade visual pós-operatório não devendo ser o fator determinante na indicação ou contraindicação do procedimento cirúrgico e sim, mais um parâmetro que deverá ser somado ao quadro clínico do paciente, tendo em vista a grande variação apresentada de resultados satisfatórios e insatisfatórios em relação à morfologia dominante da catarata e a sua hipoestimação na maioria dos casos estudados ${ }^{(10-11)}$.

\section{CONCLUSÃO}

O PAM hipoestimou ou manteve a acuidade visual na grande maioria dos casos (87\%). Ao correlacionar com a classificação morfológica dominante da opacidade do cristalino, este apresentou uma fidelidade inversamente proporcional à intensidade de opacidade do cristalino no caso da catarata nuclear com $76,5 \%$, e $60,9 \%$ quando $1+/ 4,2+/ 4$, e decresceu para 44,4\% com $3+/ 4$ e $33,3 \%$ quando $4+/ 4$; esta acurácia foi maior nos casos de catarata subcapsular posterior $(85,7 \%)$. Quando hiperestimado, houve associação com doenças oculares na maioria dos casos. 


\begin{tabular}{|l|ccc|}
\hline \multicolumn{4}{|c|}{ Tabela 3. Relação dos resultados satisfatórios com classificação } \\
morfológica dominante da catarata
\end{tabular}

\begin{tabular}{|cccc|}
\hline \multicolumn{4}{|c|}{ Tabela 4. Relação da opacificação nuclear com os resultados } \\
satisfatórios \\
$\begin{array}{c}\text { Opacificação } \\
\text { nuclear (+) }\end{array}$ & $\begin{array}{c}\text { Satisfatório } \\
\text { No olhos (\%) }\end{array}$ & $\begin{array}{l}\text { Insatisfatório } \\
\text { No olhos (\%) }\end{array}$ & $\begin{array}{c}\text { Total } \\
\text { № olhos (\%) }\end{array}$ \\
$1+$ & $13(76,5 \%)$ & $4(23,5 \%)$ & $17(100 \%)$ \\
$2+$ & $14(60,9 \%)$ & $9(39,1 \%)$ & $23(100 \%)$ \\
$3+$ & $4(44,4 \%)$ & $5(55,6 \%)$ & $9(100 \%)$ \\
$4+$ & $1(33,3 \%)$ & $2(66,7 \%)$ & $3(100 \%)$ \\
\hline
\end{tabular}

Com este conhecimento prévio, o cirurgião pode oferecer ao seu paciente uma melhor e mais real expectativa de resultados visuais pós-operatórios em cirurgias de catarata.

\section{ABSTRACT}

Purpose: To evaluate the correlation between the preoperative visual acuity (VA) obtained by the potential acuity meter (PAM) and the postoperative VA in a patient submitted to cataract surgery, as well as its correlation with the dominant morphologic classification of the cataract. Methods: This is a prospective study performed at the Hospital Monumento Study Center. Sixty-three eyes of 45 patients submitted to phacoemulsification with intraocular lens implantation were enrolled in this study and 1 eye had been excluded. Besides the complete examination in the preoperative period, PAM was used and its results were compared with the VA at the third postoperative month and correlated with the dominant morphologic cataract classification. The result was called satisfactory when the variation was equal to or less than two lines at the Snellen chart. We transformed the AV into $\log$ MAR for comparison with the literature. Results: The mean age was 45.3 years with a mean VA of $0.64 \log$ MAR by the PAM. At the third postoperative month, the mean best corrected visual acuity (BCVA) was $0.09 \log$ MAR. The PAM overestimated the BCVA in 8 eyes $(13 \%)$, underestimated it in 41 eyes $(66 \%)$ and in 13 eyes $(21 \%)$ the BCVA were the same. The satisfactory results regarding nuclear cataract were reduced in cases of higher density (1+/4 with $75.5 \%$ e $4+/ 4$ with $33.3 \%)$ and increased in the posterior subcapsular cataracts $(85.7 \%)$. Conclusion: PAM revealed an underestimation or maintenance of the BCVA in most cases $(87 \%)$. Its fidelity was inversely proportional in the nuclear cataracts (1+/4 with $75.5 \%$ e $4+/ 4$ with $33.3 \%)$ and higher in the posterior subcapsular cataracts.

Keywords: Cataract extraction; Visual acuity/physiology; Vision tests/methods; Diagnostic techniques, ophthalmological/instrumentation

\section{REFERÊNCIAS}

1. Odom JV, Chao GM, Weinstein GW. Preoperative prediction of postoperative visual acuity in patients with cataracts: a quantitative review. Doc Ophthalmol. 1988;70(1):5-17.

2. Gus PI, Kwitko I, Roehe D, Kwitko S. Potential acuity meter accuracy in cataract patients. J Cataract Refract Surg. 2000;26(8):1238-41.

3. Lasa MS, Datiles MB $3^{\text {rd }}$, Freidlin V. Potential vision tests in patients with cataracts. Ophthalmology. 1995;102(7):1007-11.

4. Minkowski JS, Palese M, Guyton DL. Potential acuity meter using a minute aerial pinhole aperture. Ophthalmology. 1983;90(11):1360-8.

5. Chylack LT Jr, Leske MC, McCarthy D, Khu P, Kashiwagi T, Sperduto R. Lens opacities classification system II (LOCS II). Arch Ophthalmol. 1989; 107(7):991-7.

6. Graney MJ, Applegate WB, Miller ST, Elam JT, Freeman JM, Wood TO, et al A clinical index for predicting visual acuity after cataract surgery. Am J Ophthalmol. 1988;105(5):460-5.

7. Miller ST, Graney MJ, Elam JT, Applegate WB, Freeman JM. Predictions of outcomes from cataract surgery in elderly persons. Ophthalmology. 1988;95(8): 1125-9.

8. Tharp A, Cantor L, Yung CW, Shoemaker J. Prospective comparison of the Heine retinometer with the Mentor Guyton-Minkowski potential acuity meter for the assessment of potential visual acuity before cataract surgery. Ophthalmic Surg. 1994;25(9):576-9.

9. Faulkner W. Laser interferometric prediction of postoperative visual acuity in patients with cataracts. Am J Ophthalmol. 1983;95(5):626-36.

10. Cuzzani OE, Ellant JP, Young PW, Gimbel HV, Rydz M. Potential acuity meter versus scanning laser ophthalmoscope to predict visual acuity in cataract patients. J Cataract Refract Surg. 1998;24(2):263-9.

11. Melki SA, Safar A, Martin J, Ivanova A, Adi M. Potential acuity pinhole: a simple method to measure potential visual acuity in patients with cataracts, comparison to potential acuity meter. Ophthalmology. 1999;106(7):1262-7. 